\title{
Innovations in Nursing and Midwifery Education and Practice: New York University College of Nursing Working with Rwandan Colleagues
}

\author{
Melissa T. Martelly ${ }^{1}$, Lydie Mukashyaka ${ }^{1}$, Glorieuse Uwizeye ${ }^{1}$, Deborah A. Chyun ${ }^{2}$, Robin T. Klar ${ }^{2}$ \\ ${ }^{1}$ Ministry of Health, Rwanda \\ ${ }^{2}$ New York University College of Nursing, NYC, NY
}

\section{Background}

New York University College of Nursing (NYUCN) is one of the original U.S. institutions to support the Rwandan Ministry of Health's initiative to provide 'mentoring and formal instruction to increase nursing and midwifery clinical and management competencies using evidence-based knowledge and practices. NYUCN's unique contribution for Human Resources for Health (HRH)-Rwanda is the capacity to hire non-U.S. faculty adding another dimension of clinical practice and expertise.

\section{Objectives}

Raise the skill level of nursing and midwifery workforce within identified clinical settings.

Support existing and new pathways for advanced nursing and midwifery education.

\section{Methods}

Recruited and hired qualified nursing and midwifery faculty. Provided direct and virtual supervision to HRH-Rwanda NYUCN faculty via annual in-country site visits, e-mail and telephonic support as needed. Conducted annual reviews of HRH-Rwanda NYUCN faculty progress and goals. Participated in monthly HRH-Rwanda coordinator and nursing and midwifery conference calls.

\section{Results}

Successful faculty recruitment and retention: Since the program's inception NYU has had 33 nursing and midwifery faculty in Rwanda.Three annual in-country visits for direct supervision with this last visit participating in the Global Innovations in Nursing and Midwifery Education, Research and Practice Conference where 9 out of 10 abstracts were accepted involving 27 nurses and midwives, $70 \%$ of which were Rwandan. Health impacts included: training in basic life support, evidence-based surgical, maternal \& neonate patient management, triage, poverty reduction, and education of Rwanda nursing and midwifery students.

\section{Conclusion}

NYUCN collaboration in the HRH-Rwanda project has demonstrated success in raising the skill level of the nursing and midwifery workforce in Rwandan clinical facilities as evidenced by the outstanding reach of health and education impacts. NYUCN has also demonstrated success in supporting the existing and future academic enterprise at the University of Rwanda School of Nursing and Midwifery. Furthermore many NYUCN faculty have chosen to enlist for a second or third year to continue the positive work completed to date.

Key words: collaborative partnerships, human resources for health, nursing 